\section{Aleksandra Paliczuk}

Università della Slesia, Katowice

Polonia

(iD https://orcid.org/0000-0002-9759-4882

\section{La concettualizzazione del verbo 'mettere' in italiano}

The Conceptualization

of the verb 'mettere' ('to put') in Italian

\begin{abstract}
The conceptualization of space is manifested in language through diverse linguistic structures. Space, one of the most significant analytical categories not only in linguistics, introduces a variety of senses and conceptual relations in the construction of communicative meaning. While there are several approaches to linguistic studies, the most obvious choice for this type of analysis seems to be Cognitive Linguistics, with some of its theoretical currents and the Cognitive Grammar of Ronald W. Langacker (1987, 1991a, 1991b, 1995, 2008) in particular. In his works, Langacker often refers to spatial and visual relationships that provide useful illustrations to depict different conceptual structures and relationships. Indeed, the relations between visual perception and conceptualization concerns numerous aspects of the semantics of natural language (E. Tabakowska, 1999: 59). The paper aims to analyse the concept of the Italian verb 'mettere' ('to put'), apparently simple and yet, as it will be shown, rich and varied in meaning.
\end{abstract}

\title{
Keywords
}

Cognitive Linguistics, imagery, linguistic picture of the world, profile, to put (It.:'mettere')

\section{Parole chiave}

Linguistica Cognitiva, l'immaginare, l'immagine linguistica del mondo, il profilo, 'mettere' 


\section{Introduzione}

Per poter analizzare la lingua in quanto strumento che serve alla comunicazione tra nazioni diverse, anzi tra culture diverse, bisogna riferirsi alle teorie linguistiche che cercano di spiegare quale sia il ruolo della lingua nel processo di conoscere il mondo, di imparare, sperimentare, percepire e concettualizzare. $\mathrm{Ci}$ sono parecchi approcci tra cui quello più adeguato per questo tipo di analisi sarà proprio la Linguistica Cognitiva con alcune sue proposte teoriche: in particolare, la grammatica cognitiva di Ronald W. Langacker, la quale propone un approccio complessivo e coerente alla lingua. Langacker (1987, 1991a, 1991b, 1995, 2008), nei suoi lavori, si riferisce spesso alle relazioni spaziali e visuali che, secondo lui, costituiscono illustrazioni utili per descrivere diverse strutture e relazioni concettuali. Infatti, la relazione tra la percezione visiva e la concettualizzazione riguarda numerosi aspetti della semantica del linguaggio naturale (E. Tabakowska, 1999: 59). Questo lavoro è un tentativo di analizzare il modo di concettualizzare il verbo italiano 'mettere', il cui significato a prima vista sembra abbastanza semplice ma, come vedremo, risulta assai ricco e variegato.

\section{I fondamenti teorici}

Il senso degli enunciati è fondato nell'interazione sociale, si verifica negli atti di comunicazione tra parlanti ed è correlato con la nostra interpretazione del mondo, vuol dire la sua immagine mentale che poi viene rappresentata con le unità linguistiche.

Nell'ambito degli studi cognitivi sulla lingua si fa riferimento agli studi di Edward Sapir (1978) in cui si considera il rapporto tra la lingua e l'esperienza del mondo. Sapir (1978) parla della lingua come di uno strumento che permette di descrivere la realtà, l'esperienza e le interpretazioni individuali del mondo che, però, raggiungono una comprensione comune. Secondo Sapir, le forme linguistiche determinano certi modi di osservazione e di interpretazione. La lingua ci aiuta e allo stesso tempo ci disturba nelle nostre esperienze conoscitive e a ciò sono dovute alcune sottili differenze semantiche tra diverse culture. Grazie alla continua interazione e penetrazione tra lingua e esperienza, lo status della lingua come sistema non è puramente simbolico, ha anche un carattere contestuale. Anzi, la lingua non soltanto rinvia alla nostra esperienza del mondo, ma la può formare ed interpretare o scoprire nel senso che il parlare e l'agire si intrecciano continuamente e si completano l'uno con l'altro nelle nostre attività ed interazioni quotidiane (E. Sapir, 1978: 38-40). 
In questo lavoro si fa particolare ricorso alla nozione di immaginare, che fa parte della grammatica cognitiva di Langacker (2008). Secondo lui, la grammatica come tale, per mezzo dei suoi elementi, porta con sé il significato (le parole) e permette di costruire e simboleggiare significati più sofisticati, nascosti negli enunciati complessi quali i sintagmi o le frasi (cf. A. Paliczuk, 2016: 221). La grammatica non soltanto costituisce una parte integrante dei processi cognitivi, ma è una chiave per la loro comprensione (R.W. Langacker, 2008: 17-18). L'oggetto di studio della grammatica cognitiva è la concettualizzazione in correlazione con le espressioni linguistiche. Langacker (1999) dice che il significato di un'espressione linguistica non si limita soltanto al contenuto concettuale a cui si riferisce, ma è anche costituito dal processo dell'immaginare convenzionale (conventional immagery) o della costruzione della scena (scene construal) (cf. A. Paliczuk, A. Pastucha-Blin, 2016: 144). Il processo dell'immaginare (o della costruzione della scena) si basa essenzialmente sulla percezione dello spazio e anche in altre teorie nel campo della linguistica cognitiva si registra il riferimento terminologico allo spazio e alla sua visione, come p.e.: l'immagine, l'osservatore, il punto di vista, la prospettiva, l'organizzazione 'figura-sfondo', la traiettoria, gli spazi mentali ecc. Non sorprende, quindi, il fatto che l'uomo ne approfitti nei processi cognitivi e, di conseguenza, nell'uso quotidiano della lingua.

Nel campo degli studi linguistici polacchi, Jerzy Bartmiński introduce la nozione di Jęykowy Obraz Świata (JOS), che si potrebbe tradurre come l'immagine linguistica del mondo - la nozione è legata alla visione del mondo (in inglese view of the world) e all'immagine del mondo (in tedesco das sprachliche Weltbild). La visione implica il fatto di vedere, guardare, osservare, allora gli elementi del processo sono: a) il soggetto guardante, cioè la persona che guarda, percepisce qualcosa, e b) l'immagine - in quanto il risultato del processo di vedere il mondo da parte di qualcuno (l'immagine e la visione del mondo non sono però nozioni equivalenti) (J. Bartmiński, 1999: 103). Invece, l'immagine linguistica del mondo è un'interpretazione della realtà contenuta nella lingua; si basa sulle opinioni del mondo radicate nella lingua in strutture grammaticali, forme lessicali, modi di dire, proverbi, testi (J. Bartmiński, 1999: 104). Nelle analisi linguistiche si dovrebbe considerare dunque il rapporto tra la lingua e la realtà circostante, vale a dire che la lingua è lo strumento che serve ad interpretare il mondo, non lo crea e non lo riflette. L'immagine linguistica del mondo è una struttura concettuale, caratteristica per ogni lingua, per mezzo della quale la gente comprende (percepisce e poi interpreta, categorizza) il mondo. È una struttura soggettiva, però abbastanza universale o caratterizzata da certe somiglianze dentro un gruppo di persone che usano la stessa lingua (o anche lingue diverse), perciò la comunicazione e la comprensione reciproca sono possibili (R. Grze-

${ }^{1}$ Sull'immaginare e sul profilare nella lingua ci sono già parecchi lavori nell'ambito della linguistica cognitiva anche tra i linguisti polacchi (J. Bartmiński, 1993 e molti altri lavori: A. Kosz, 2005, 2006, 2008, A. Paliczuk, 2014, 2016, A. Paliczuk e A. Pastucha-Blin, 2016, 2019 ed altri). 
gorczykowa, 1999: 45-46). È quindi un'interpretazione del mondo verbalizzata in modi diversi (J. Bartmiński, 2009: 12).

In ambedue gli approcci, di Langacker e di Bartmiński, appare la nozione di profilare; è il processo mentale che avviene nella relazione tra la nostra percezione e concettualizzazione della realtà ed il modo in cui ne parliamo. La lingua serve da strumento grazie a cui siamo in grado di trasmettere informazioni, messaggi, nostre opinioni, credenze, impressioni ed infatti interpretazioni del mondo reale e di quello astratto. Il profilare nella teoria di Langacker è una delle dimensioni dell'immaginare, ossia il processo mentale grazie a cui possiamo creare nuovi concetti, nuovi significati, possiamo manipolare gli elementi del nostro sistema concettuale per formulare enunciati in modo da trasmettere nel modo più preciso possibile la nostra visione del mondo. Secondo Bartmiński (2009: 89-90), il profilo è un particolare (anche soggettivo) modo di comprensione di un oggetto. I profili di un concetto non sono i suoi nuovi significati, ma sono i modi di organizzare i contenuti semantici nell'ambito del suo significato di base.

Questo lavoro si basa sulla nozione di profilare in quanto processo di creazione di nuove sfumature di significato (o meglio nuovi sensi) e riguarda l'analisi del verbo 'mettere' da diversi punti di vista, diverse prospettive. Si cercherà di analizzare alcuni contesti in cui il verbo in questione appare, per dimostrare quali significati può assumere.

Il profilare consiste nello scegliere un elemento dalla base cognitiva e sottolinearlo per palesare il significato che vogliamo applicare in un dato enunciato parlando di un determinato frammento della realtà, sia quella fisica sia quella astratta. Si vedranno dunque moltissimi esempi di espressioni e modi di dire in cui 'mettere' assume diversi nuovi significati in relazione al contesto e alla situazione in cui si trova. 'Mettere' è universale e generico, ma allo stesso tempo presenta una ricchezza di significati in diversi contesti, vale a dire che potrebbe essere spiegato in altre lingue con numerosi lessemi diversi (p.e. in polacco: położyć, włożyć, nałożyć, ułożyć, założyć, kłaść, układać, wkładać, nakładać, zakładać, poukładać... ecc. e sono soltanto gli esempi del significato di base).

La parte successiva del testo costituisce un tentativo di analisi dei profili di 'mettere', eseguita in base alla nozione di profilare, grazie a cui si può conoscere la varietà dei significati e dei contesti nei quali il verbo appare e quali accezioni assume. 


\section{L'analisi dei profili di 'mettere'}

Osservando la definizione del verbo mettere presa dal dizionario online 'Treccani' $^{2}$, si può spiegare il suo significato nel modo seguente:

Verbo di sign. ampio e generico, dai confini semantici non ben definiti, che comprende in sé le accezioni di porre, collocare, posare, introdurre, ficcare, attaccare, versare, e di parecchi altri verbi, da cui può di volta in volta essere opportunamente sostituito. Regge per lo più due complementi, il compl. oggetto e un compl. di luogo o di termine, e sono questi che ne determinano caso per caso il significato. 1. In genere, far stare in un luogo, far passare da un posto a un altro, sia spostando l'oggetto con le mani sia in altro modo; $m$. in, dentro, sopra, sotto, accanto, davanti, dietro; $m$. il tappeto in terra, $i$ vestiti nell'armadio, i libri nello zaino; $m$. i piatti, le posate, le vivande in tavola (spesso ellitticamente: $m$. in tavola); $m$. le merci in magazzino, le casse in deposito, la vettura in rimessa, un'imbarcazione in mare; $m$. le botti sul carro; $m$. la legna nel caminetto; $m$. i panni in lavatrice; $m$. i fagioli in pentola, la carne sulla griglia, la minestra sul fuoco; $m$. l'acqua nel bicchiere, lo zucchero nel caffe, il vino in bottiglia; $m$. il cappello in testa; $[. .$.$] (https://www.treccani.it/vocabo-$ lario/mettere/ data accesso: 20.10.2020).

Volendo semplificarla, si potrebbe dire che 'mettere' significa far così che un oggetto occupi una determinata posizione o un determinato luogo. Sarà dal suo significato di base che nascono molti altri. Infatti, il suo significato dipende dalla situazione comunicativa e dal contesto in cui viene collocato, assumendo così diverse sfumature semantiche e accezioni specifiche, vuol dire estensioni metaforiche.

Si possono osservare delle analogie tra la percezione dello spazio fisico e quello astratto, vale a dire che il verbo 'mettere' si applica a entrambi i casi: alla descrizione dello spazio fisico e alla descrizione di quello astratto. Il significato del verbo dunque si attualizza nella concettualizzazione delle azioni astratte in cui diversi concetti vengono trattati tramite il prisma della realtà fisica. Con i significati di porre, collocare, posare e così via, il verbo appare in numerosi contesti linguistici che riflettono il modo di percepire e concettualizzare la realtà circostante, un modo che viene poi trasmesso alla concettualizzazione delle nozioni astratte. Quindi, si possono manipolare gli oggetti fisici grazie alla varietà di usi possibili del verbo 'mettere' per poi trasmetterli alla manipolazione dei concetti astratti.

${ }^{2}$ Tutte le definizioni delle espressioni analizzate sono prese dai dizionari online: https://www. treccani.it/, https://dizionari.corriere.it/, https://dizionari.repubblica.it/italiano.html, https://www. garzantilinguistica.it/. Anche spiegazioni ed esempi riportati nel testo sono creati in base alle voci trovate in questi dizionari. 
Nei paragrafi che seguono si tenta di evidenziare le accezioni del verbo 'mettere' raggruppandole in profili e sottoprofili a seconda dei significati che rappresentano le locuzioni citate.

Si propone una classificazione di profili e sottoprofili, tra cui si distinguono due gruppi generali: il profilo di spazio astratto (con i sottoprofili: di contenitore e di superficie) e i profili basati sulle parti del corpo, cioè i profili di spostamento, di intervento, di intenzione, di pensiero, di fiducia, di azione ed il profilo emotivo.

\subsection{Il profilo di spazio astratto}

Nel presente paragrafo vengono analizzati gli esempi in cui si approfitta della manipolazione degli oggetti nella realtà fisica per poter parlare dei fenomeni astratti. Vediamo l'esempio:

\section{(1) Questo thriller mette i brividi!}

Siccome i brividi non sono un oggetto reale, ma una sensazione, si ha a che fare in questo caso con l'analogia tra il fisico e l'astratto; nella locuzione mettere $i$ brividi, quell'altro oggetto o una superficie su cui li si mettono è sottintesa. La manipolazione delle astrazioni nel nostro sistema concettuale si conferma in numerose espressioni, p.e.:

(2) Obama aveva messo la pace mondiale al primo punto del suo programma.

(3) L'insegnante cerca di mettere pace tra due allievi che litigano.

Dunque, si può mettere (la) pace in un posto, al primo punto, tra le persone ecc. come se la pace fosse un oggetto, di conseguenza il verbo 'mettere' assume un senso figurato.

Un altro esempio in cui si vede l'analogia tra la percezione di oggetti situati nello spazio fisico e in quello astratto è:

\section{(4) L'hanno messo sotto accusa.}

L'espressione 'mettere sotto accusa' risulta dalla situazione in cui qualcuno mette un oggetto sotto un altro oggetto, p.e. come mettere sotto il coperchio, mettere sotto l'ombrellone e così via. Ė evidente, quindi, analizzando gli esempi sopraccitati con il verbo 'mettere', che la percezione e la concettualizzazione del modo di manipolare oggetti concreti, reali, ha un grande impatto sul modo in cui vengono concettualizzate le azioni mentali svolte sulle nozioni astratte; ciò si evidenzia tramite le strutture lessicali italiane. 


\subsubsection{Il sottoprofilo di contenitore}

La maggior parte delle espressioni in cui appare il verbo 'mettere' si riferisce alla percezione e alla concettualizzazione di oggetti concreti e poi astratti, durante le quali gli uni e gli altri vengono collocati in un tipo di contenitore - sia quello fisico che quello figurato. Si usano in italiano, ad esempio, le locuzioni in cui abbiamo a che fare proprio con i contenitori figurati, come p.e.:

(5) Ne ho abbastanza di sentirti mettere in dubbio ogni mia parola.

(6) Non vorrei essere costretto a mettere in pericolo le persone a cui tengo.

(7) Non era la mia intenzione di mettere in imbarazzo chi non conosce la risposta.

Il dubbio, il pericolo, l'imbarazzo, ecc. sono concetti astratti, tuttavia quell'unione del verbo 'mettere' con astrazioni mediante la preposizione 'in' indica che le percepiamo e concettualizziamo come contenitori (basandoci sulle espressioni come: mettere in una scatola, in una pentola, in un armadio ecc.). Si noti un altro esempio:

(8) Hai messo troppe idee nel tuo discorso.

Anche in questo caso si può parlare della concettualizzazione del discorso in quanto contenitore, e di conseguenza, il verbo 'mettere' assume un senso astratto - diventa un'azione mentale.

\subsubsection{Il sottoprofilo di superficie}

Possiamo notare molte espressioni in cui le preposizioni indicano il tipo di relazione che esiste tra gli elementi di un sintagma; così si verifica l'azione di mettere un oggetto su una superficie, come p.e.: mettere il francobollo ad una lettera, mettere le corde al violino, mettere un quadro alla parete ecc. La stessa relazione, introdotta da 'mettere' e preposizione 'a', riguarda spesso le azioni sui concetti astratti, p.e.: mettere qc. a disposizione, a rumore, a profitto, mettere al corrente, mettere al mondo e altri. Vediamo alcuni esempi:

(9) Il comune ha messo a disposizione un ampio parcheggio nel centro della città.

(10) È un fatto che ha messo a rumore tutta la città.

(11) In materia di gestione del rischio occorre mettere a profitto le esperienze acquisite da altri Paesi.

(12) Giulia mi ha messo al corrente della situazione. 
(13) È emozionante, mettere al mondo un bambino.

(14) Hanno finalmente messo il titolo all'opera.

Negli esempi sopraccitati vediamo la trasposizione del significato di 'mettere qc. a qc.', vale a dire che si tratta dell'immagine di una situazione in cui mettiamo un oggetto a/su o presso un altro, lo aggiungiamo ad un altro, lo mettiamo su una superficie ecc., come, ad esempio, quando mettiamo un quadro ad una parete, oppure come mettiamo qc. (o qu.) al sole, all'aria, ossia cambiamo le condizioni, le circostanze, in cui un oggetto si trova. Si possono dunque notare gli usi di altre preposizioni, come 'su' o 'in', nelle espressioni con 'mettere' in senso astratto, p.e.:

(15) Hai sbagliato, però qualcuno ti può ancora mettere sulla via giusta (sulla strada giusta, sulla retta via $\neq$ sbagliata).

(16) Dio ti ha messo sulla mia strada.

La prima locuzione significa indirizzare qualcuno nel suo ragionamento o nel suo comportamento, dargli indicazioni su come agire o pensare - l'espressione si basa sull'analogia di come indirizziamo qu. dirigendolo verso un luogo (fisico); invece nella seconda frase abbiamo l'espressione basata sulla metafora della VITA COME STRADA (o STRADE), vale a dire che nella vita arriviamo a conoscere diverse persone così come, passeggiando per le strade, incontriamo diversa gente. Inoltre, entrambe le locuzioni si basano sul senso di 'mettere qc. su qc.' come nel caso di mettere le botti sul carro, mettere la carne sulla griglia ecc. Pensando all'immagine della strada come percorso non soltanto fisico, ma anche mentale, riguardante pure le azioni mentali, si noti un altro modo di dire:

(17) Lui ha messo degli ostacoli ${ }^{3}$ sul tuo sentiero e ora devi riuscirci a risolvere i tuoi problemi.

(18) Hanno messo gli ostacoli nel vostro cammino per cercare di fermarvi.

(19) La Russia ha messo ostacoli nella modalità di controllo delle elezioni.

I primi due esempi rinviano in modo evidente alla metafora menzionata (della strada, via, del percorso come modo di vivere, agire), il terzo è ancora più astratto: abbiamo la modalità di fare qc. in quanto modo di procedere, vuol dire che le difficoltà nella realizzazione di un'azione sono come ostacoli incontrati camminando per strada.

In italiano interessanti sono le locuzioni: mettere su casa o mettere su famiglia, che vediamo negli esempi che seguono:

3 'Mettere gli /degli ostacoli' sul sentiero o nel cammino di qu. sono esempi che potrebbero entrare anche nel profilo di intervento per il suo senso che riguarda le interazioni, tuttavia, l'espressione è priva di parti del corpo. 
(20) Mia madre vorrebbe che io mi sistemassi già e mettessi su casa.

(21) Pensa a sposarti e a metter su famiglia.

In ambedue i casi si nota il verbo 'mettere' con la preposizione 'su' e qualcosa di sottinteso su cui si mette un oggetto, qui proprio la casa o la famiglia.

\subsection{I profili basati sulle parti del corpo}

Entrando proprio nei particolari dei diversi contesti in cui appare il verbo 'mettere' si possono notare numerose espressioni con i riferimenti alle parti del corpo umano. La gente 'mette le parti del corpo' in diversi luoghi o posizioni per esprimere determinati significati. Questo fenomeno può essere spiegato con un modo generale di percepire la realtà circostante, vuol dire nella maggior parte delle lingue si osserva un forte antropocentrismo nel modo di conoscere il mondo (riflesso poi nella lingua): parlando della realtà l'uomo spesso fa riferimento a se stesso, si tratta come punto di riferimento intorno a cui avvengono azioni, eventi, hanno luogo situazioni. E dato che conosciamo il mondo prima con i sensi, dunque impariamo come manipolare gli oggetti fisici, quando, poi, giungiamo a comprendere le astrazioni, negli anni successivi del nostro sviluppo, allora impariamo a manipolare le astrazioni in base alla nostra esperienza empirica.

Nei paragrafi che seguono vengono evidenziati diversi esempi in cui per mezzo del verbo 'mettere' si esprimono vari comportamenti, atteggiamenti, azioni, anche o innanzitutto quelli che riguardano relazioni interpersonali, ma pure quelli che si riferiscono al modo di provare emozioni (profilo emotivo). Ne sono stati distinti alcuni sottoprofili (di intervento, di intenzione, di pensiero, di fiducia, di azione ed emotivo) in cui vengono analizzate le locuzioni riguardanti diversi comportamenti umani.

\subsubsection{Il profilo di spostamento}

Il primo profilo evidenziato nel gruppo delle espressioni che rinviano alle parti del corpo è il profilo di spostamento. Per quanto riguarda il movimento o lo spostamento sembra naturale l'uso delle parti del corpo, tuttavia si possono osservare le locuzioni in cui appaiono altre parti del corpo (non sempre i piedi o le gambe) che non necessariamente implicano il movimento. Vediamo l'esempio:

(22) Oggi non ho messo il naso fuori.

Analizzando il significato della frase sopraccitata si ha in mente l'immagine di una persona che sta per uscire attraverso la porta, sta sulla soglia con i piedi, e la 
parte del corpo che è la più esterna e sporge è proprio il naso. In conseguenza, viene usato il naso messo fuori (o no) per dire che qualcuno esce di casa (o no si usa l'espressione piuttosto per dire che qualcuno non è uscito).

Una delle locuzioni che impegna il piede per indicare lo spostamento è:

\section{(23) A casa tua non metterò più piede!}

'Mettere piede' in un posto, in un luogo significa venirci. La persona, camminando per spostarsi, usa ambedue i piedi, ambedue le gambe e infatti tutto il corpo si sposta.

In questi due casi (esempio 22 e 23) si ha a che fare con la metonimia (parte per tutto) in cui il naso o il piede rappresentano tutta la persona.

Il modo di muoversi, e infatti di comportarsi, che caratterizza una persona, viene contenuto in:

\section{(24) Non ti mettere sempre tra i piedi!}

'Mettersi tra i piedi' è una locuzione che descrive l'azione di impedire a qu. di camminare, farlo inciampare, e viene usata per parlare di una persona imbarazzata, impacciata che camminando infastidisce gli altri.

\subsubsection{Il profilo di intervento}

Nel presente sottocapitolo si analizzano le locuzioni in cui 'mettere' assume il significato del verbo che indica l'azione di intervenire, intromettersi nelle azioni o negli affari altrui. L'espressione che chiude il paragrafo precedente, 'mettersi tra i piedi', può essere analizzata anche da un altro punto di vista, p.e.:

(25) E c'è sempre la suocera a mettersi tra i piedi dei giovani.

La si usa, allora, anche per descrivere la situazione nella quale uno si immischia, si intromette negli affari altrui per impedirgli di fare qc. (come si può impedire un cammino fluente facendo così che qu. cada e non possa andare avanti). Quindi, in base all'immagine della situazione in cui qualcuno mette qualcosa tra i piedi di qualcuno per farlo cadere, si ha a che fare con la trasposizione del significato concreto in quello astratto, vuol dire l'espressione assume il senso di ostacolare qualcuno in un'azione (qualsiasi).

L'esempio successivo riguarda la situazione comunicativa in cui qualcuno interviene:

(26) Non mettere bocca (lingua) in un discorso degli adulti! 
La bocca (la lingua) viene associata all'attività di parlare, pronunciare qc.; allora questa parte del corpo rappresenta qui parole, idee, opinioni pronunciate da qualcuno. In questo caso il significato dell'espressione sarà spiegato come: intromettersi, entrare a parlare senza essere chiamati, senza essere interrogati, siccome mettendo la bocca in un discorso si pronunciano parole o enunciati non richiesti dall'altra persona e li si introducono in quel discorso interrompendo l'enunciato di qualcun altro.

Vediamo un'altra espressione:

\section{(27) Non mettere il naso negli affari altrui!}

È un esempio dell'espressione che rappresenta un comportamento inappropriato, vuol dire: intromettersi, impicciarsi di cose che non ci riguardano. La locuzione si basa sulla concettualizzazione del naso come rappresentante dell'olfatto, vale a dire che quando uno vuole trovare qualcosa, spiando, a volte usa l'olfatto per sentire l'odore di qualche cosa che lo porterà a quello che cerca.

\subsubsection{Il profilo di intenzione}

In italiano si possono incontrare delle locuzioni che contengono il verbo 'mettere' in combinazione con le parti del corpo che esprimono un'intenzione di chi parla, ad esempio:

(28) Questi vogliono solo che tutti gli mettano gli occhi addosso.

'Mettere gli occhi addosso a qu./qc.' significa guardare qualcuno / qualcosa fissamente, in particolare con l'intenzione di sedurre (qu.). Dunque, si tratta di una situazione nella quale uno vuole che tutti/tutte lo guardino, lo ammirino mettendogli gli occhi addosso così come se fossero oggetti, pezzi di gioielleria, abbigliamento e simili. La persona che 'mette gli occhi addosso a qualcuno' ha intenzione di fare qc. o di ottenere qualche risultato, di provocare un certo comportamento di un'altra persona, di fare un'impressione ecc.

Un senso piuttosto negativo assume la locuzione seguente:

(29) Mai mettere le mani addosso ad una ragazza.

(30) Per una sciocchezza mi ha messo le mani addosso e mi ha criticato di fronte a tutti.

'Mettere le mani addosso a qu.', accanto al significato proprio dell'espressione in quanto alzare le mani, picchiare o malmenare nel senso fisico, può indicare anche il maltrattamento psichico, vuol dire: criticare con severità anche eccessiva o in- 
giustificata, interpretare in maniera inesatta o forzata, minacciare qu.. In questo caso si parla di atteggiamento e intenzioni negative verso qualcuno.

Un esempio che tocca situazioni simili, vuol dire relazioni interpersonali negative, sono le locuzioni:

(31) Non fatevi mettere sotto i piedi di nessuno!

(32) Non fatevi mettere i piedi di nessuno in testa!

'Mettere i piedi in testa a qu.' oppure 'mettere qu. sotto i piedi' significa sottometterlo con prepotenza, maltrattarlo, farlo o tenerlo come schiavo, opprimerlo. Le espressioni si basano sull'immagine di uno che applica la forza fisica ad un altro usando i piedi per tenerlo.

\subsubsection{Il profilo di pensiero}

Per quanto riguarda la comunicazione di certe informazioni, idee, opinioni osserviamo i modi di dire che seguono:

(33) Sua madre le ha messo idee stravaganti in testa.

(34) Mia figlia si è messa in testa un'idea che diventerà una cantante famosa.

Nel caso delle locuzioni 'mettere qc. in testa a qu.' e 'mettersi qc. in testa' si tratta del fatto di convincere qu. (o rispettivamente: se stesso), far credere o spingere a fare qualcosa; ciò nel primo esempio si verifica tra due persone (una persona vuole convincere un'altra) e nel secondo riguarda lo stesso agente, cioè significa convincere se stessi nel senso fissare una cosa, un'idea e simili nella coscienza o nella memoria, fissarsi in un pensiero, in un proposito, incaponirsi a credere in $q c$. ecc.. Si tratta qui infatti della combinazione delle mani (sottointese, che agiscono: prendono, manipolano qualcosa) e della testa, in cui l'ultima fa da rappresentante del nostro pensare, ragionare, vuol dire è il contenitore per pensieri, idee, intenzioni, desideri e così via.

\subsubsection{Il profilo di fiducia}

Nelle relazioni sociali bisogna spesso fidarsi di altre persone e quindi anche in questo contesto usiamo le locuzioni con 'mettere' e 'le mani' per parlarne:

(35) Lei si è messa totalmente nelle mani del suo fidanzato. 
In questa frase si osserva la trasposizione dell'immagine di uno che dà qualcosa di prezioso ad un altro, perché ha fiducia di questa persona, dunque 'mettere se stessi (o qc.) nelle mani di qualcuno' significa affidarsi a lui completamente, essere sotto la sua protezione, ma anche dipendenti dalle sue decisioni. Esistono anche variazioni di quest'espressione: 'mettere qc. in buone / cattive mani', ciò significa affidare qc. ad una persona rispettivamente buona o cattiva.

L'espressione che segue è un altro esempio che combina i due concetti, 'mettere' e 'la mano':

(36) Per te metto la mano sul fuoco.

(37) Metto la mano sul fuoco che arriverà in ritardo anche questa volta.

È un modo di dire che esprime la sicurezza, la fiducia. Qualcuno afferma qualcosa in modo deciso oppure garantisce per qualcun altro con la massima sicurezza. Viene usata in questo caso l'immagine della persona che essendo tanto sicura di sé mette la mano sul fuoco, come nel caso della medievale prova del fuoco.

\subsubsection{Il profilo di azione}

Le parti del corpo servono spesso a descrivere diverse azioni, attività, modi di procedere, di iniziare un'azione o di comportarsi. Partendo dal significato di base del verbo 'mettere', come nel caso di: metter mano a qualche cosa, che indica un'azione di: afferrarla per servirsene a un determinato scopo, troviamo le sue analogie in descrizioni di diverse situazioni, anche quelle figurate, p.e.:

(38) Dobbiamo assolutamente mettere mano a una profonda riforma dei mercati finanziari.

'Mettere mano a qc.' è una locuzione che si riferisce a varie forme dell'operare umano; ad esempio: mettere mano a un lavoro significa iniziare a lavorare; mettere mano alla penna, ai pennelli, vuol dire iniziare a scrivere, a dipingere ecc.. Dunque, il suo significato sarà spiegato come iniziare $a$, cominciare a fare $q c$. E per analogia si può osservare l'esempio successivo:

(39) Dobbiamo mettere mano al portafoglio come si deve.

In questo caso il portafoglio suggerisce il significato finanziario, vuol dire si tratta dell'azione di pagare, spendere $i$ soldi, sborsare. Un'espressione simile, 'mettere mano al portafoglio di qu.' oppure 'mettere le mani sui soldi di qu.' significa invece cercare di impadronirsi dei soldi di qualcun altro. 
Un'analogia al movimento nella realtà fisica è osservabile in:

(40) La ditta ha messo le mani sulle informazioni confidenziali dell'azienda concorrenziale.

La locuzione 'mettere le mani su qc.' potrebbe considerare sia l'azione sugli oggetti fisici, concreti, che su quelli astratti. Tuttavia, in ambedue i casi, il significato è: entrare in possesso, impadronirsi di qc. o semplicemente di prendere qc.

Le mani rappresentano spesso diverse azioni siccome con le mani le eseguiamo, e così nell'esempio che segue possiamo osservare:

(41) Suo padre era un ricco mafioso e gli aveva insegnato a mettere le mani in pasta dappertutto.

(42) Ho messo le mani in una faccenda.

La prima delle due locuzioni sopraccitate significa: essere coinvolto in qc., in particolare in qc. di negativo, in affari sporchi; invece l'altro esempio non ha piuttosto una sfumatura negativa e significa prendere parte in qc., occuparsene in maniera diretta o indiretta. Si tratta dell'intromettersi, ingerirsi o del coinvolgersi in qualche cosa (per analogia di mettere le mani in un contenitore con una sostanza per fare qc. con o di essa).

Per quanto riguarda la descrizione dei modi di agire, proseguire, l'esempio successivo riguarda un'altra parte del corpo, il piede, e viene usato in riferimento a chi cammina:

(43) Questa volta ha messo il piede in fallo.

'Mettere il (un) piede in fallo' va spiegato come posarlo su terreno malsicuro, cedevole, con il significato di sbagliare, commettere un errore. Un passo sbagliato nel nostro cammino risulta in una caduta, così un piede messo in fallo in un procedimento qualsiasi indica che qu. ha sbagliato. L'espressione successiva, invece, ci permette di evitare gli errori, p.e.:

(44) I ricercatori mettono le mani avanti avvertendo di non essere sicuri che queste associazioni significhino, attualmente, che bere caffè faccia vivere più a lungo.

La locuzione si basa sull'immagine di qualcuno che prima di cadere mette le mani avanti per proteggersi dalla caduta oppure si protegge da qualcuno che lo attacca. Il suo significato figurato è: prevenire situazioni difficili o spiacevoli, garantirsi o esigere garanzie contro eventuali danni, fare patti che mettano al sicuro dal pericolo di sorprese spiacevoli. Si può prevenire un attacco fisico e quello psichico, 
mentale, come accusa, rimprovero, obiezione ecc. accusando gli altri o presentando in anticipo elementi a propria discolpa.

\subsubsection{Il profilo emotivo}

In italiano si usa un'espressione, tra molte altre, che coinvolge il verbo 'mettere' e le parti del corpo anche per esprimere emozioni, come la disperazione; vediamo l'esempio:

(45) In questi ultimi giorni, alcuni si mettono le mani nei capelli quando sentono parlare delle nuove iniziative assunte dal governo.

Si tratta di un gesto simbolico fatto quando uno si dispera, ma la locuzione viene pure usata in modo figurato (senza dover eseguire quel gesto), in quanto è un modo di dire che, per analogia con il gesto che accompagna uno stato d'animo di disperazione, effettivamente lo esprime rievocando proprio l'immagine della situazione in cui uno esegue quel gesto.

\section{Conclusioni}

$\mathrm{Ci}$ sono numerosissimi esempi delle locuzioni in cui il verbo 'mettere' assume diversi significati, molto spesso figurati, che è impossibile citarli tutti, e anche in un campione rappresentativo come quello riportato nel presente testo, è risultato difficile raggrupparli in un modo omogeneo, siccome in molti casi una data espressione potrebbe essere categorizzata in due o più profili a seconda dell'aspetto che si sottolinea e si prende in analisi. Si potrebbero creare numerosi profili o categorie in base ai diversi significati che il verbo assume in locuzioni diverse. Nell'analisi svolta sono stati individuati alcuni aspetti semantici di 'mettere' in italiano che riguardano soprattutto l'analogia nel percepire e nel concepire la realtà fisica e quella astratta (il profilo di spazio astratto); vuol dire che troviamo moltissime espressioni nelle quali, tramite le strutture lessicali, si evidenzia proprio il modo di concettualizzare le azioni mentali come fisiche. Si traspone il senso di base del verbo di operare sugli oggetti reali all'operare sulle astrazioni. È stato anche analizzato un campione di espressioni nelle quali, accanto all'analogia menzionata sopra, viene aggiunto l'uso dei concetti che descrivono le parti del corpo (i profili basati sulle parti del corpo), però non soltanto per parlare del corpo come tale e delle sue attività o azioni; in molti casi tali espressioni evidenziano le azioni mentali che si riferiscono alle interazioni o relazioni sociali (il profilo di 
intervento, il profilo di fiducia, il profilo di intenzione), allo spostarsi (il profilo di spostamento), all'agire, al procedere o al comportarsi (il profilo di azione) in qualche modo. 'Mettere' attualizza anche il senso di azione mentale (il profilo di pensiero) o di stato emotivo (il profilo emotivo). Si potrebbe concludere con l'osservazione che molto spesso i concetti che a prima vista sembrano semplici, poco complicati, quelli che non dimostrano un'evidente corrispondenza con la realtà astratta, risultano molto più complessi nel loro significato o piuttosto nei significati che la gente attribuisce ad essi. I parlanti italiani per quanto concerne il verbo 'mettere' trovano sicuramente nel suo uso quotidiano nella lingua tanti più significati e tante più espressioni in cui non solo si riflette l'analogia tra la percezione del mondo reale e quello astratto, ma soprattutto si creano contesti in cui il senso di 'mettere' è molto più sviluppato ed evoluto. L'analisi del concetto di 'mettere' 'mettere' è un tentativo di spiegare ed evidenziare come le persone, in questo caso gli italiani, percepiscono e concepiscono il mondo, lo concettualizzano e ne parlano.

\section{Riferimenti bibliografici}

Bartmiński, J. (1993). O profilowaniu i profilach raz jeszcze. In J. Bartmiński \& R. Tokarski (Red.), O definicjach i definiowaniu (s. 269-275). Lublin, Wydawnictwo Uniwersytetu Marii Curie-Skłodowskiej.

Bartmiński, J. (1999). Punkt widzenia, perspektywa, językowy obraz świata. In J. Bartmiński (Red.), Językowy obraz świata (s. 103-120). Lublin, Wydawnictwo Uniwersytetu Marii Curie-Skłodowskiej.

Bartmiński, J. (2009). Językowe podstawy obrazu świata. Lublin, Wydawnictwo Uniwersytetu Marii Curie-Skłodowskiej.

Grzegorczykowa, R. (1999). Pojęcie językowego obrazu świata. In J. Bartmiński (Red.), Językowy obraz świata (s. 39-46). Lublin, Wydawnictwo Uniwersytetu Marii Curie-Skłodowskiej.

Kosz, A. (2005). Occhio all'italiana - cioè l'immagine linguistica del mondo italiano. Neophilologica, 17, 177-186.

Kosz, A. (2006). L'immaginare. I profili dell'occhio nelle lingue: italiana, polacca ed inglese. Linguistica Silesiana, 27, 105-115.

Kosz, A. (2008). Il passo dal pensiero alla lingua - l'analisi cognitiva della STRADA nella lingua italiana. Neophilologica, 20, 124-141.

Langacker, R. W. (1982). Space Grammar, Analysability, and English Passive. Language, $58,22-80$.

Langacker, R. W. (1987). Foundations of Cognitive Grammar. Theoretical Prerequisites (Vol. 1). Standford, Standford University Press.

Langacker, R. W. (1991a). Concept, Image, And Symbol. The Cognitive Basis of Grammar. Berlin — New York, De Gruyter Mouton. 
Langacker, R. W. (1991b). Foundations of Cognitive Grammar. Descriptive Application (Vol. 2). Standford, Standford University Press.

Langacker, R. W. (1995). Wykłady z gramatyki kognitywnej (H. Kardela, P. Łozowski, Przeł.). Lublin, Wydawnictwo Uniwersytetu Marii Curie-Skłodowskiej.

Langacker, R. W. (1999). Grammar and Conceptualization. Berlin - New York, De Gruyter Mouton.

Langacker, R. W. (2008). Cognitive Grammar. A Basic Introduction. Oxford, Oxford University Press.

Langacker, R. W. (2009). Gramatyka Kognitywna. Wprowadzenie (E. Tabakowska, M. Buchta, H. Kardela et al., Przeł.). Kraków, Universitas.

Paliczuk, A. (2014). Spazio - pensiero - lingua. La concettualizzazione della 'città' in italiano". Neophilologica, 26, 298-309.

Paliczuk, A. (2016). Paese che vai usanze che trovi. La concettualizzazione del "paese" in italiano. Neophilologica, 28, 220-231.

Paliczuk, A., \& Pastucha-Blin, A. (2016). Il concetto di "porta" nel discorso italiano. Linguistica Silesiana, 37, 143-159.

Paliczuk, A., \& Pastucha-Blin, A. (2019). La doppia natura dell'acqua nei testi indirizzati alle donne. Kwartalnik Neofilologiczny, LXVI(4), 610-624.

Sapir, E. (1978). Kultura, język, osobowość. Wybrane eseje. Warszawa, Państwowy Instytut Wydawniczy.

Tabakowska, E. (1999). Gramatyka i obrazowanie. Wprowadzenie do językoznawstwa kognitywnego. Kraków, PAN „Nauka dla wszystkich”.

\section{Dizionari online}

https://dizionari.corriere.it/

https://dizionari.repubblica.it/italiano.html

https://www.garzantilinguistica.it/

https://www.treccani.it/ 